REVIEW ARTICLE

OPEN

\title{
Super-enhancer function and its application in cancer
} targeted therapy

\author{
Faqing Tang $\mathbb{B}^{1,3 凶}$, Zongbei Yang ${ }^{2,3}$, Yuan $\operatorname{Tan}^{1}$ and Yuejin $\mathrm{Li}^{1}$
}

Recently, super-enhancers (SEs) have been identified as a unique type of transcriptional regulation involved in cancer development. SEs exhibit a size, high transcription factor density, and strong binding to the transcriptional machinery compared with typical enhancers. SEs play an essential role in cell growth, differentiation, and disease initiation and progression including tumorigenesis. In particular, cancer-specific SEs have been proven to be key oncogenic drivers types of tumor cells. Furthermore, it has been confirmed that cancer-specific SEs can mediate the dysregulation of signaling pathways and promote cancer cell growth. Additionally, therapeutic strategies directly targeting SE components, for example, by disrupting SE structure or inhibiting SE cofactors, have shown a good curative effect on various cancers.

npj Precision Oncology (2020)4:2 ; https://doi.org/10.1038/s41698-020-0108-z

\section{INTRODUCTION}

In eukaryotic cells, transcription begins with RNA polymerase binding to the promoters of DNA molecules, and transcription is regulated by transcription factors (TFs) through binding to specific DNA sequences to recruit RNA polymerase II initiation or elongation factors. ${ }^{1}$ The promoter region harbors transcription initiation sites. Additionally, there are some DNA sequences located near or far from promoter regions that contain multiple transcription factor binding sites. These DNA sequences are referred to as "enhancers", and they increase gene expression. ${ }^{2,3}$ Recently, with the development of high throughput sequencing, increasing numbers of enhancers have been detected on a genome-wide scale. A coactivator that was proven to be an enhancer increases target gene expression. ${ }^{4}$ The coactivator does not bind DNA, but it pairs with TFs, to further activate gene transcription. The coactivator has general activation domains that facilitate its interaction with the basal transcription or chromatin remodeling machinery. ${ }^{5}$ Additionally, the histone modification of H3K4 trimethylation has been found to be associated with active promoters. ${ }^{6}$ DNase hypersensitive sites partially overlap with enhancer regions, and enhancer activation coincides with the DNase I hypersensitivity of these regions, which is often associated with specific posttranslational modifications of adjacent nucleosomes. ${ }^{7-10}$ Direct interaction or looping between enhancers and promoters has been observed and might be critical for enhancer function. ${ }^{11,12}$ Super-enhancers (SEs) defined as clusters of enhancers in close genomic proximity are flanked by CTCF (CCCTC-binding factor) binding sites, and are involved in regulating the expression of key genes. ${ }^{13}$

Gene transcriptional dysregulation, which is one of the core tenets of cancer development, ${ }^{14}$ involves in noncoding regulatory elements, including TFs, promoters, enhancers, SEs, and RNA polymerase II (Pol II). ${ }^{15}$ In particular, SEs have been found to play core roles in promoting oncogenic transcription to accelerate cancer progression. ${ }^{16,17}$ Herein, we introduce the concept, function and identification of SEs, and summarize the contribution of oncogenic SEs to cancer and the challenges of SEs in therapy.
This will assist us to in achieving a profound understanding of the functional mechanism by which SEs regulate target gene expression.

\section{SES DEFINITION AND GENE TRANSCRIPT CONCEPT}

TFs are proteins that bind DNA helices at specific regulatory sequences to activate or inhibit gene transcription through a trans-activation or trans-repression domain. TFs locate their target sequences and unlock the pathway for subsequent functions, such as transcription, replication, and repair. ${ }^{18}$ TFs bind to and activate enhancers to initiate gene transcription. ${ }^{8,19}$ Enhancers are defined as short ( 100-1000 bp) noncoding DNA sequences, composed of concentrated clusters of TF recognition motifs. Enhancers drive gene transcription independent of their distance, location or orientation relative to their cognate promoter. ${ }^{19,20} \mathrm{SEs}$ are a large cluster of transcriptional enhancers that have been proposed to consist of a long genomic domain composed of an enhancer cluster occupied by high levels of H3K4me1, H3K27ac, p300 or master TFs. SEs span a range of more than $20 \mathrm{~kb}$ on average. They differ from classical enhancers in their size, transcription factor density and content, sensitivity of binding to perturbation, and active transcription (Fig. 1). SEs produce higher levels of enhancer RNAs (eRNAs) than enhancers, ${ }^{13}$ and present high potential to activate the transcription of target genes and drive the expression of genes. ${ }^{21}$ SEs are defined as follows: the identification of SEs is based on the differences in their ability to bind markers of promoter transcriptional activity, including cofactors (such as mediators and cohesins), histone modification markers (such as $\mathrm{H} 3 \mathrm{~K} 27 \mathrm{ac}$ and H3K4me1), and chromatin modification molecules (such as p300) (Fig. 2).

Recently, "stretch-enhancers" were similar to SEs; stretchenhancers induce high expression levels of target genes. ${ }^{13,21-23}$ A stretch-enhancer is a region of $\geq 3 \mathrm{~kb}$ according to the ChromHMM algorithm, that displays an enhancer chromatin state. ${ }^{23,24} \mathrm{SE}^{\prime}$ regions are identified by binding with mediator protein (MED) 1, the enrichment of histone H3K27 acetylation (H3K27ac), or binding with cell-type-specific TFs. ${ }^{25}$ SEs are

\footnotetext{
${ }^{1}$ Department of Clinical Laboratory, Hunan Cancer Hospital and the Affiliated Cancer Hospital of Xiangya School of Medicine, Central South University, 410013 Changsha, China. ${ }^{2}$ Department of Clinical Laboratory, Zhuhai People's Hospital \& Zhuhai Hospital of Jinan University, 519000 Zhuhai, China.. ${ }^{3}$ These authors contributed equally: Faqing Tang, Zongbei Yang ${ }^{凶}$ email: tangfaqing33@hotmail.com
} 


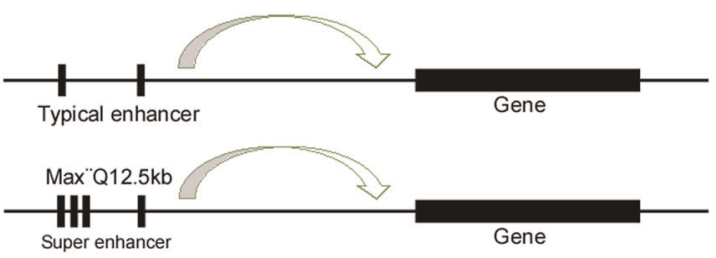

Fig. 1 Schematic structure of typical enhancer and super-enhancer.

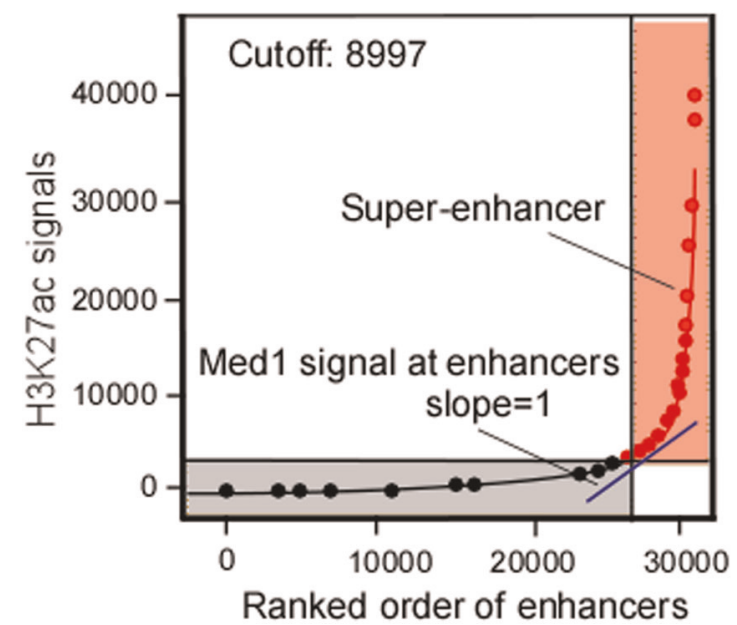

Fig. 2 H3K27ac signal within $\mathbf{1 2 . 5}$ kb window at significant peak were ranked for enhancers. Peak with four times higher H3K27ac signals than the rest of the peak were assigned as superenhancer (SE).

predicted to be located in bioinformatic stitching regions within $12.5 \mathrm{~kb}$ in the linear genome. As a result, SEs are longer than traditional enhancers, and often contain more than one separate region that is bound by multiple TFs. ${ }^{13,21,22}$

There are still some controversies about SE characterization. Since SEs were initially characterized by Whyte and Loven in $2013,{ }^{13,22}$ a number of publications have referred to "superenhancers", although SE identification has been inconsistent. In some reports, the definition of "super-enhancer" deviates from specific properties of SEs such as chromatin marks and cofactors bound to these regions. In other studies, enhancers and SEs are not defined functionally, and enhancer stitching is an algorithmic step, not a selection criterion. The only defining feature of a "super-enhancer" is an exceptionally high degree of enrichment of transcriptional activators or chromatin marks, as determined by chromatin immunoprecipitation sequencing (ChIP-seq). ${ }^{26}$ Use of the term "enhancer" has also shifted from a functional definition of a DNA element that activates gene transcription to a looser definition based on chromatin profiles, characterized by DNAse I hypersensitivity, p300 binding, or H3K4me, or H3K27ac marks. The recent explosion of large-scale genomic data has led many researchers to carefully revisit these concepts.

\section{SE IDENTIFICATION}

For identification of SEs in mouse embryonic stem cells (mESCs), the active enhancer site was identified, and the binding sites of key TFs, Oct4, Sox2 and Nanog (OSN) binding sites were then sequenced via ChIP-seq analysis ${ }^{13,21}$; "constituent" enhancers were identified as a class of regulatory regions with unusually strong enrichment, all of which were merged into a large region (Fig. 1). Thereafter, the stitched enhancers were ranked by the normalized level of the Med1 signal in the genomic region, and the curve of the signal to rank plot was generated. A line with a slope of 1 tangent to the curve was used as a cutoff point to separate SEs and typical enhancers (TP), where a position above the line indicated an SE, and a position below the line indicated a TP (Fig. 2). Third, mESC enhancers were divided into two classes based on mediator levels, with one class encompassing the vast majority of enhancers, and the other containing 231 large enhancer domains. Approximately $40 \%$ of the Med1-binding signal was found to localize to SEs spanning DNA regions whose median length was an order of magnitude larger than that of a typical enhancer. The large enhancers constituted less than $3 \%$ of the total enhancer regions across the genome in terms of region and the density of binding. ${ }^{13}$ Some reports have shown that the co-occupancy of ESC genomic sites by OSN TFs is highly predictive of enhancer activity. 4,27

A total of 8794 enhancers were identified in ESCs by using ChIPseq data sets. In the differentiated cells, lineage-specific master regulators were used to generate binding plots of the OSN master TFs. ${ }^{13}$ SEs in mESCs were identified using key TFs such as PU.1, MyoD, T-bet, and C/EBPa from myotube cells, pro-B cells, Th cells and macrophages, and master TFs in these cells were found to be associated with SEs. ${ }^{13}$ SEs have been defined in many cells and tissues by tissue-specific master TFs; however, the master TFs constituting SEs are still not known, and genome-wide binding data are still limited. The various surrogate marks of typical enhancers include H3K27ac, H3K4me1, p300 and DNase, and the histone $\mathrm{H} 3 \mathrm{~K} 27 \mathrm{ac}$ modification is superior to the others in mESC. ${ }^{4,6,28-31}$ A catalog of super-enhancers has been generated for 86 human cell and tissue types, and these SEs have been associated with genes based on cell-type-specific TFs. Thus, candidate master TFs have been identified in many cell types, which has proven to be useful for better understanding the transcriptional control of the cell state and reprogramming. ${ }^{21}$ SEs and their potential applications are increasingly being recognized and cited, and these findings have been widely used for the exploration of disease mechanisms, such as those of tumors. ${ }^{22,32-}$ 35 Additionally, human $\mathrm{H} 1$ embryonic stem (H1ES) cells were found to contain 6426 stretch-enhancers (with a mean size of 4434 bp) $)^{23}$ and 684 SEs. $^{21}$ Among the 684 SEs, 505 overlap with a stretch-enhancer. According to the common definitions of SEs, the number of stretch-enhancers exceeds that of SEs by an order of magnitude. Thus, SEs are a subset of stretch-enhancers, and the two entities are not interchangeable as defined.

\section{SE STRUCTURE AND FUNCTION}

Functional characteristics of SEs

Compared with other enhancers, SEs exhibit stronger transcriptional activation and a stronger regulatory ability for the genes that they control. ${ }^{13}$ Some researchers have cloned DNA fragments from SE elements into luciferase reporter vector, and then transfected the vector into ESCS to determine SE functionality. Constituent enhancer segments within SEs are defined as 600-1400 bp regions with a single peak of OSN occupancy, that generate higher luciferase activity than typical enhancer segments (3.8-fold high). SEs show a stronger ability to drive target gene transcription than typical enhancers. ${ }^{36}$ Interestingly, the functional interactions of SE constituents are neither superimposed nor synergistic, indicating that one component has complex effects on the activity of another component. Some SEs show increased effects on transcriptional activity, and some have inhibitory effects. ${ }^{36}$ SEs exhibit complicated interdependence on each other's activity, following optimal transcriptional activity. SEs also present master transcription factor-dependent characteristics, exhibiting cell-type-specific functions and producing strong responses. In ESCs, a reduction in Oct4 leads to the loss of ESC- 
specific gene expression and differentiation. If SE-associated genes are more sensitive to the loss of master TFs than other genes, a reduction in Oct4 level should cause preferential loss of SE-associated gene expression. Upon knockdown of the transcription factor Oct4 in mESCs, the cells lose their pluripotent state. ${ }^{13}$ In this process, the expression of SE-correlated genes is decreased, and their expression is lower than that of typical enhancer related genes. These results indicate that SEs exhibit a higher interference sensitivity than TEs. ${ }^{11,13,22,37}$ When mediator levels are reduced using small hairpin RNAs (shRNAs), the greatest effects on gene expression were observed for SE-associated genes. ${ }^{13,37,38}$ If SEassociated genes are more sensitive to coactivator loss than other genes, a reduction in the levels of mediator subunits should preferentially affect the expression of SE-associated genes. Reduced levels of mediators and cohesins have the same effect on the key characteristics of the ES cell state as the loss of Oct4 itself. $^{38}$

\section{Structural characteristics of SEs}

SEs drive the expression of cell-specific genes, and are densely occupied by the transcription apparatus and its cofactors including cohesins. ${ }^{11,30}$ The cohesin-mediated substructure of gene loops, such as cohesin-associated enhancer-promoter loops and cohesin-associated CTCF loops, regulates gene expression (Fig. 3). The analysis of high-confidence cohesin ChIA-PET (Chromatin Interaction Analysis using Paired End Tag sequencing, ChIA-PET) interaction data revealed striking features common to loci containing SEs and their associated genes. These features consist of SEs and the associated genes located within a loop, which are connected by two interacting CTCF sites co-occupied by cohesin. A total of $84 \%$ of ESC-SEs are contained within these structures, whereas $48 \%$ of typical enhancers occur within comparable loops between two CTCF sites. ${ }^{39}$ Usually, one SE only contains one domain, and SEs are restricted to activate genes in SDs (Fig. 3). The loss of this restriction is likely to result in inappropriate activation of neighboring genes, which are incorrectly targeted frequently enough to cause tumors to occur. ${ }^{39,40}$ In mESC, SEs were defined as clusters of enhancers occupied by OSN and other mediators; in addition, CHIP-seq analysis of the terminal TFs of the Wnt, TGF and LIF signaling pathways showed that SEs

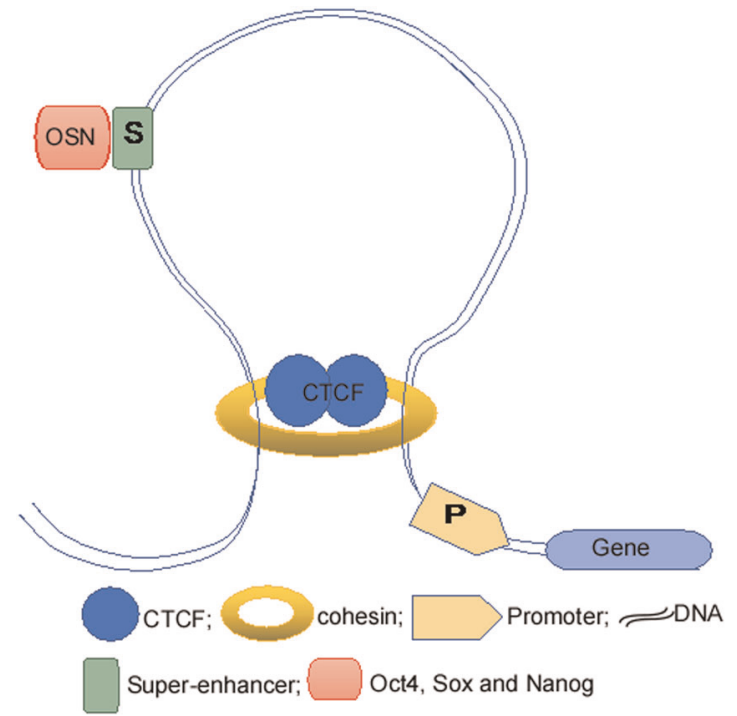

Fig. 3 Schematic illustration of super-enhancer-mediated gene expression. Cohesin-mediated substructure of gene loops, cohesinassociated enhancer-promoter loop and cohesin-associated CTCF loop regulates gene expression. CTCF CCCTC binding factor, P promoter, OSN Oct4, Sox and Nanog. bound to individual enhancers with a similar pattern to OSN. ${ }^{36,41,42}$ SEs can determine cell identity as a platform for dense transcription factor binding associated with different signaling pathways. ${ }^{36}$ eRNAs are a type of noncoding RNAs (ncRNAs) that are transcribed and synthesized from the enhancer regions of genome. eRNA plays a stimulatory role in the transcription of related genes, and exhibits a genome-wide character. ${ }^{43}$ eRNAs serve as a "linker" between SEs and their target genes. The coordinated regulation of transcripts by eRNA can be plotted in active RNA Pol II global nuclear run-on sequencing (GRO-seq) experiments. The transcriptional and synthetic eRNAs of enhancers are conducive to the function of enhancers, and their transcriptional levels are correlated with high activity of enhancer. ${ }^{44,45}$ eRNA has been studied in various cell types including neurons, macrophages, $\mathrm{T}$ cells and cancer cells, ${ }^{44,46-48}$ and approximately $30.6 \%$ of typical enhancers and $93.3 \%$ of SEs overlap with eRNAs in intergenic regions. In response to Toll-like receptor signaling in macrophages, SE-related genes and eRNA show coordinated changes. However, these signaldependent SEs, which are associated with innate immunity and inflammation, are different from the ones identified on the basis of cellular characteristics. ${ }^{45}$

\section{SE' FUNCTIONS IN CANCER DEVELOPMENT AND APPLICATIONS IN CANCER THERAPY}

Oncogenic SEs activate oncogenic signaling

Compared with TEs, SEs are enriched in one or more binding sites for the terminal TFs of signaling pathways and signaling modules, and function in the maintenance of stemness and pluripotency. Extending this principle, oncogenic SEs are rich in TF binding sites, and associated with specific signaling pathways upon which cancer cells depend. ${ }^{36}$ Oncogenic SEs promote tumorigenesis and malignancy by upregulating oncogene transcription. ${ }^{21}$ Mechanistically, oncogenic SEs may activate the MAPK signaling pathway to inhibit apoptosis and promote the proliferation of cancer cells. ${ }^{49}$ SEs also induce overexpression of the $v$-ets erythroblastosis virus E26 oncogene homolog (ERG), leading to the overexpression of target genes driving the development of cancer. ${ }^{50}$ Additionally, oncogenic SEs upregulate CYP24A1, GJA5, SLAMF7, and ETV1 in squamous cell carcinoma. ${ }^{51}$ The translocation of SEs upregulates MYB expression in adenoid cystic carcinoma (ACC), and SEs promote the expression of TERT in pheochromocytomas and paragangliomas. $^{52}$

Cancer cells frequently acquire SEs to promote oncogene expression, which mediates the dysregulation of signaling pathways. $^{21,33,35,53}$ Colorectal cancer (CRC) is driven by various oncogenic pathways, ${ }^{14}$ and CRC-associated SEs are enriched in transcription factor 4 (TCF4) binding sites. ${ }^{36}$ CRC-associated SEs are found in CRC cells but not in normal colon cells, indicating that these SEs are specific to CRC tumorigenesis. The analysis of the ChIP-seq binding profile of CRC cells shows that TCF4 is a terminal TF of the Wnt pathway and occupies at the c-MYC locus (Table 1). TCF4 is a well-established target of Wnt signaling that shows strong H3K27Ac signal after cancer cells acquire oncogenic SEs. ${ }^{36}$ Acquired SE-associated genes are enriched after the stimulation or blockage of the Wnt pathway, but not all SE genes display this response. This observation supports the notion that the acquired SEs may be dominated by TCF4 and may respond to perturbation of the oncogenic Wnt pathway. ${ }^{36}$ H3K27Ac ChIP-seq analysis of estrogen receptor (ESR)-positive McF-7 cells indicate that the SE of the ESR1 gene only encodes estrogen receptor alpha (ERa) in tumor cells. Furthermore, oncogenic transcription can distinguish cancer subtypes relying on distinct signaling pathways (Table 1). In ER-positive breast cancer cells, SE-associated genes are enriched for ERa binding, whereas in triple-negative breast cancer cells, SE enriched sites are different from those of oncogenic TFs. ${ }^{21,54}$ One 
Table 1. SEs involved in cancer.

\begin{tabular}{llllc}
\hline TF binding with SE & Target molecular/signal pathway & Tumor type & Tumorigenesis & Therapy \\
\hline TCF4 & C-MYC/Wnt & Colorectal cancer & $\sqrt{ }$ \\
ESR & ERA/Channel oncogenic signal pathway & Breast cancer & $\sqrt{ }$ & 35 \\
BRD4 & C-MYC & Unascertained & $\sqrt{ }$ & 20,51 \\
TAL1 & MYB & Leukemia & $\sqrt{ }$ & $\sqrt{ }$ \\
GATA2 & AVI1 & Neuroblastoma & $\sqrt{ }$ \\
GATA3 & LMO1 & Lung adenocarcinoma & $\sqrt{ }$ & 34 \\
NFE2L2, CEBPB & MYC & Adenold cystic carcinoma & $\sqrt{ }$ \\
MYB/TGFBR3 & MYB/RAD51B & Vi, & 33 \\
\hline
\end{tabular}

$A M L$ acute myeloid leukemia, BRD4 bromodomain-containing protein 4, ERA estrogen receptor alpha, ESR estrogen receptor, EVI1 ecotropic virus integration site-1, SE super-enhancer, TAL1 T-cell acute lymphoblastic leukemia transcription factor 1, TCF4 transcription factor 4, TF transcription factor, TGFBR3 transforming growth factor beta receptor III.

of the general functions of SEs may be to channel oncogenic signaling pathways into gene expression programs, which is required for sustaining cancer development. ${ }^{36}$

Chromatin regulators are regulated by an SE inhibitor

SEs were found to be associated with tumorigenesis in a myeloma cell line by Loven et al. ${ }^{22}$ Chromatin regulators are attractive therapeutic targets for cancer due to their deregulation in numerous cancers, 55,56 and are regulated by an SE smallmolecule inhibitor. ${ }^{57,58}$ The inhibition of some chromatin regulators has been proven to be efficacious for cancer treatment. ${ }^{59}$ Many reports focus on cancer treatment through inhibiting the expression of chromatin regulators, and inhibitors of chromatin regulators have been used to selectively inhibit the transcription of key oncogenic drivers in multiple ways. Most chromatin regulators are expressed in a broad range of normal cells, and exhibit an adverse effect on global gene expression. The small molecule JQ1 (BET bromodomain inhibitor) can selectively repress MYC expression by decreasing bromodomain-containing protein 4 (BRD4) binding to c-MYC SE regions. ${ }^{2,60-63}$ BRD4 is a member of the bromodomain and extraterminal (BET) subfamily of human bromodomain proteins, which is associated with acetylated chromatin and involved in transcriptional activation. ${ }^{64,65}$ It recruits positive transcription elongation factor $b$ ( $P$-TEFb) to regulate transcriptional elongation by RNA Pol II. ${ }^{66,67}$ BRD4 displays similar binding patterns to mediators, localizing to regulatory regions of the actively transcribed genes, especially at SEs. Its inhibition mediates the preferential loss of BRD4, which results in a corresponding decrease in MED1 (mediator of RNA polymerase II transcription subunit 1) binding and transcription (Table 1). Cancer cells "acquire" specific SEs near oncogenes, which occurs in a gene desert near c-MYC but is absent in healthy cells. This acquisition of specific SEs is thought to contribute to tumorigenesis. The key oncogenic drivers of tumor cells are regulated by SEs, which can confer disproportionate sensitivity to BRD4 coactivator loss and cause selective inhibition of transcription. ${ }^{21}$ This functional characteristic of cancer cell SEs may be used to identify key oncogenes and develop target drugs. ${ }^{22,68,69}$

\section{Mechanisms of oncogenic SE formation}

Cancer cells acquire cancer-specific SEs that are not present in their normal counterparts. DNA translocation, transcription factor overexpression, and focal amplification frequently occur in cancer, and these changes may result from cancer cells acquiring SEs. Overexpression of TAL1 (T-cell acute lymphoblastic leukemia transcription factor, TAL1) in a subset of acute lymphoblastic leukemia (ALL) is associated with SE formation. TAL1 upstream of an SE contains a short heterozygous somatic mutation that creates one SE and introduces binding motifs for the MYB transcription factor in noncoding sites ${ }^{35}$ (Table 1). MYB binding with the SE generates a positive feedback loop that reinforces its own expression, which activates an MYB-dependent oncogenic transcriptional program ${ }^{40,53,70,71}$ (Table 1).

Insertion mutations, chromosomal inversions and translocations play a central role in the pathogenesis of almost all cancers. A distal enhancer of the GATA2 gene arising upon chromosomal $3 q$ rearrangement ectopically activates EVI1 (ecotropic virus integration site-1) expression, which leads to the concomitant loss of GATA2 transcription. ${ }^{40}$ The loss of GATA2 is associated with acute myeloid leukemia (AML), and GATA2 haploid in sufficiency might provide the precise context for EVI1-mediated oncogenic transformation $^{40,72,73}$ (Table 1). In addition to the above cases, single nucleotide polymorphisms also have a direct effect on the regulation of oncogenic SEs. In neuroblastoma, GATA3 plays a central role in regulating $L M O 1$ expression. SE formation at the LMO1 oncogene depends on GATA protein3 binding at the conserved intronic GATA. Knockdown of GATA3 results in a decrease in LMO1 and suppression of cell growth. The protective T allele (TATA) disrupts GATA3 binding, and leads to reduced recruitment of H3K27Ac, which is negatively associated with the LMO1 SE in neuroblastoma cells ${ }^{34}$ (Table 1). Focal amplification of SEs can also result in aberrant oncogene expression. In lung adenocarcinoma, the focal amplification of $\sim 450 \mathrm{~kb}$ downstream of the MYC locus leads to SE formation, and drives high expression of the oncogene, in which NFE2L2 and CEBPB are necessary to maintain SE activity. ${ }^{74}$ A chromosomal translocation in ACC repositions an unrelated SE in proximity to the MYB oncogene, resulting in high MYB expression ${ }^{75}$ (Table 1 ).

Therapeutic strategy of targeting oncogenic SEs

Since cancer cells exhibit increases in the level of oncogenic transcriptional activity and growth-promoting pathways, a specific therapeutic strategy targeting oncogenic transcription has been developed. ${ }^{16,76}$ Inhibiting oncogenic transcription is an attractive therapeutic option; however, transcription is a biological process that is fundamental to all living cells, and inhibiting transcription has dire consequences for cell gene expression ${ }^{16,77}$; thus, any clinically useful transcriptional inhibitor should selectively target oncogenic transcription with only minimal toxicity in normal cells. JQ1, iBET, and bromodomain inhibitors selectively bind to the domains of BRD4, ${ }^{78,79}$ which causes selective repression of the MYC oncogene in a wide range of tumors including multiple myeloma (MM), Burkitt's lymphoma (BL), AML, and $\mathrm{ALL}^{60-63,80}$ (Table 2). SE-associated transcription depends on the cooperative binding of BRD4 and mediators, and the recruitment of the CDK7containing initiation complex (TFIIH) and CDK9-containing elongation complex (p-TEFb). JQ1 is a competitive inhibitor of BRD4 that 
Table 2. Therapeutic SEs and inhibitors.

\begin{tabular}{lllll}
\hline SE inhibitor & Molecular target & Function & Ref. \\
\hline JQ1 & BRD4 & Reducing BRD4 occupancy and MED1 binding, Pol II stalling and elongation impairment & AML \\
IBET & CDKs & Regulating RNAPII initiation and elongation & Unascertained & 81 \\
THZ1 & CDK7 & Inhibiting phosphorylation of CTD of RNA Pol II and hindering promoter proximal pausing & Neuroblastoma & 65 \\
& MYCN & Repression of MYCN-dependent transcriptional amplification & Neuroblastoma & 65 \\
\hline
\end{tabular}

AML acute myeloid leukemia, BRD4 bromodomain-containing protein 4, CDK cyclin-dependent kinase, CTD carboxyl-terminal domain, Pol II polymerase II.

works by reducing BRD4 occupancy and reducing levels of MED1 binding, resulting in Pol II stalling and impaired elongation. Such an SE-driven transcriptional program is mainly dependent on BRD4; it is important to maintain oncogenic identity and pluripotency. ${ }^{76,81}$ iBET, which is similar to JQ1, has also been applied for cancer treatment. ${ }^{82}$ In addition to BET inhibitors, targeting CDKs to regulate RNAPII initiation and elongation shows great potential ${ }^{83}$ (Table 2 ).

Recent studies have demonstrated that covalent inhibitors of CDK7 and 12 electively kill cancer cells by inhibiting SE-driven oncogenic transcription, but lack systemic toxic effects in vivo. ${ }^{76,81}$ THZ1 is a highly specific covalent inhibitor of CDK7, and CDK7 is known to promote transcription activation through phosphorylation of RNA Pol II; THZ1 inhibits the phosphorylation of the carboxyl-terminal domain (CTD) of RNA Pol II and hinders promoter proximal pausing. ${ }^{84}$ Because SEs are enriched at the paused RNA Pol II, THZ1-induced deficiency at the pause sites leads to the decreased occupancy of Pol II at these enhancers, culminating in transcriptional inhibition ${ }^{85,86}$ (Table 2). The enrichment of master TFs in SEs maintains gene expression through autoregulatory feed-forward loops, and their depletion may lead to the inhibition of transcriptional output. The MYC oncoprotein has been verified to stimulate tumor cell growth and proliferation through the amplification of gene transcription; therefore, inhibiting MYC function might be an attractive therapeutic option. It has been demonstrated that genomic amplification of the MYCN oncogene by promoting SEs causes upregulation of the active transcriptional program of neuroblastoma (NB) cells and sensitizes NB cells to the inhibition of CDK7. When a covalent inhibitor of CDK7 is used to disrupt the transcription of amplified MYCN in NB cells, the oncoproteins are downregulated with the consequent strong suppression of MYCN. THZ1 selectively targets MYCNamplified NB cells, and THZ1 target treatment leads to the preferential downregulation of SE-associated genes and significantly represses MYCN-dependent transcriptional amplification ${ }^{68}$ (Table 2). The requirement of tumor cell for the high expression of oncogenes contributes to their vulnerability to super-enhancer disruption. Some SE inhibitors cause preferential loss of SEregulated elements and TFs at SE-associated genes, for example BRD4 inhibition leads to the preferential disruption of SEs. SEs occupied by BRD4 regulate critical oncogene expression in MM, which shows that BRD4 inhibition leads to preferential disruption of these super-enhancers. This preferential disruption of SE function may be a general approach for selectively inhibiting the oncogenic drivers of cancer cells. ${ }^{22}$

\section{CONCLUSION}

SEs are large clusters of transcriptional enhancers that drive gene expression to control cell identity. Compared with normal enhancers, SEs display a unique structure and strong functional properties. Although there is still a lack of uniform rules for the definition of SEs, the differences in transcription factor density and content, binding sensitivity, and active transcription are used to distinguish SEs from ordinary enhancer, ${ }^{13}$ and whether this can become a comprehensive concept remains to be further studied and verified. ${ }^{26}$ SEs have been proven to be valuable in pathologic studies of disease. SEs are key regulators of the expression of key oncogenes in many tumor cells. Some reports have shown that SEs can promote oncogene overexpression, and disrupting SE structure and inhibiting cofactors may be specific routes for cancer therapy. Recent studies have demonstrated that some inhibitors (such as JQ1 and CDK7) selectively kill cancer cells by inhibiting SE-driven oncogene transcription. However, transcription is a fundamental biological process shared by all living cells, and targeting transcription may therefore have dire consequences for global gene expression. Along with the development of SE research, there may be novel ideas about therapeutic techniques for diseases such as cancer and other complex diseases.

Received: 12 May 2019; Accepted: 31 December 2019; Published online: 12 February 2020

\section{REFERENCES}

1. Kim, T. K. \& Shiekhattar, R. Architectural and functional commonalities between enhancers and promoters. Cell 162, 948-959 (2015).

2. Banerji, J., Rusconi, S. \& Schaffner, W. Expression of a beta-globin gene is enhanced by remote SV40 DNA sequences. Cell 27, 299-308 (1981).

3. Grosveld, F., van Assendelft, G. B., Greaves, D. R. \& Kollias, G. Position-independent, high-level expression of the human beta-globin gene in transgenic mice. Cell 51, 975-985 (1987).

4. Visel, A., Rubin, E. M. \& Pennacchio, L. A. Genomic views of distant-acting enhancers. Nature 461, 199-205 (2009).

5. Pobbati, A. V. \& Hong, W. Emerging roles of TEAD transcription factors and its coactivators in cancers. Cancer Biol. Ther. 14, 390-398 (2013).

6. Heintzman, N. D. et al. Distinct and predictive chromatin signatures of transcriptional promoters and enhancers in the human genome. Nat. Genet. 39, 311-318 (2007)

7. Lelli, K. M., Slattery, M. \& Mann, R. S. Disentangling the many layers of eukaryotic transcriptional regulation. Annu. Rev. Genet. 46, 43-68 (2012).

8. Spitz, F. \& Furlong, E. E. Transcription factors: from enhancer binding to developmental control. Nat. Rev. Genet. 13, 613-626 (2012).

9. Bulger, M. \& Groudine, M. Functional and mechanistic diversity of distal transcription enhancers. Cell 144, 327-339 (2011).

10. Shlyueva, D., Stampfel, G. \& Stark, A. Transcriptional enhancers: from properties to genome-wide predictions. Nat. Rev. Genet. 15, 272-286 (2014).

11. Deng, W. et al. Controlling long-range genomic interactions at a native locus by targeted tethering of a looping factor. Cell 149, 1233-1244 (2012).

12. Tolhuis, B., Palstra, R. J., Splinter, E., Grosveld, F. \& de Laat, W. Looping and interaction between hypersensitive sites in the active beta-globin locus. Mol. Cell 10, 1453-1465 (2002)

13. Whyte, W. A. et al. Master transcription factors and mediator establish superenhancers at key cell identity genes. Cell 153, 307-319 (2013).

14. Lu, J. et al. MICAL2 mediates p53 ubiquitin degradation through oxidating p53 methionine 40 and 160 and promotes colorectal cancer malignance. Theranostics 8, 5289-5306 (2018).

15. Sengupta, S. \& George, R. E. Super-enhancer-driven transcriptional dependencies in cancer. Trends Cancer 3, 269-281 (2017).

16. Bradner, J. E., Hnisz, D. \& Young, R. A. Transcriptional addiction in cancer. Cell 168 629-643 (2017). 
17. Lee, T. I. \& Young, R. A. Transcriptional regulation and its misregulation in disease. Cell 152, 1237-1251 (2013).

18. Zaret, K. S. \& Carroll, J. S. Pioneer transcription factors: establishing competence for gene expression. Genes Dev. 25, 2227-2241 (2011).

19. Buecker, C. \& Wysocka, J. Enhancers as information integration hubs in development: lessons from genomics. Trends Genet.: TIG 28, 276-284 (2012).

20. Schaffner, W. Enhancers, enhancers-from their discovery to today's universe of transcription enhancers. Biol. Chem. 396, 311-327 (2015).

21. Hnisz, D. et al. Super-enhancers in the control of cell identity and disease. Cell 155, 934-947 (2013).

22. Loven, J. et al. Selective inhibition of tumor oncogenes by disruption of superenhancers. Cell 153, 320-334 (2013).

23. Parker, S. C. et al. Chromatin stretch enhancer states drive cell-specific gene regulation and harbor human disease risk variants. Proc. Natl Acad. Sci. USA 110, 17921-17926 (2013).

24. Ernst, J. et al. Mapping and analysis of chromatin state dynamics in nine human cell types. Nature 473, 43-49 (2011).

25. Khan, A. \& Zhang, X. dbSUPER: a database of super-enhancers in mouse and human genome. Nucleic Acids Res. 44, D164-D171 (2016).

26. Pott, S. \& Lieb, J. D. What are super-enhancers? Nat. Genet. 47, 8-12 (2015).

27. Chen, $X$. et al. Integration of external signaling pathways with the core transcriptional network in embryonic stem cells. Cell 133, 1106-1117 (2008).

28. Rada-Iglesias, A. et al. A unique chromatin signature uncovers early developmental enhancers in humans. Nature 470, 279-283 (2011).

29. Visel, A. et al. ChIP-seq accurately predicts tissue-specific activity of enhancers. Nature 457, 854-858 (2009).

30. Creyghton, M. P. et al. Histone H3K27ac separates active from poised enhancers and predicts developmental state. Proc. Natl Acad. Sci. USA 107, 21931-21936 (2010).

31. Neph, S. et al. An expansive human regulatory lexicon encoded in transcription factor footprints. Nature 489, 83-90 (2012).

32. Vaharautio, A. \& Taipale, J. Cancer. Cancer by super-enhancer. Science $\mathbf{3 4 6}$, 1291-1292 (2014).

33. Chapuy, B. et al. Discovery and characterization of super-enhancer-associated dependencies in diffuse large B cell lymphoma. Cancer Cell 24, 777-790 (2013).

34. Oldridge, D. A. et al. Genetic predisposition to neuroblastoma mediated by a LMO1 super-enhancer polymorphism. Nature 528, 418-421 (2015).

35. Mansour, M. R. et al. Oncogene regulation. An oncogenic super-enhancer formed through somatic mutation of a noncoding intergenic element. Science 346, 1373-1377 (2014).

36. Hnisz, D. et al. Convergence of developmental and oncogenic signaling pathways at transcriptional super-enhancers. Mol. Cell 58, 362-370 (2015).

37. Niwa, H. et al. Interaction between Oct3/4 and Cdx2 determines trophectoderm differentiation. Cell 123, 917-929 (2005).

38. Kagey, M. H. et al. Mediator and cohesin connect gene expression and chromatin architecture. Nature 467, 430-435 (2010).

39. Dowen, J. M. et al. Control of cell identity genes occurs in insulated neighborhoods in mammalian chromosomes. Cell 159, 374-387 (2014).

40. Groschel, S. et al. A single oncogenic enhancer rearrangement causes concomitant EVI1 and GATA2 deregulation in leukemia. Cell 157, 369-381 (2014).

41. Ng, H. H. \& Surani, M. A. The transcriptional and signalling networks of pluripotency. Nat. Cell Biol. 13, 490-496 (2011).

42. Young, R. A. Control of the embryonic stem cell state. Cell 144, 940-954 (2011).

43. Kim, T. K. et al. Widespread transcription at neuronal activity-regulated enhancers. Nature 465, 182-187 (2010)

44. Lam, M. T., Li, W., Rosenfeld, M. G. \& Glass, C. K. Enhancer RNAs and regulated transcriptional programs. Trends Biochem. Sci. 39, 170-182 (2014).

45. Hah, N. et al. Inflammation-sensitive super enhancers form domains of coordinately regulated enhancer RNAs. Proc. Natl Acad. Sci. USA 112, E297-E302 (2015).

46. De Santa, F. et al. A large fraction of extragenic RNA pol II transcription sites overlap enhancers. PLOS Biol. 8, e1000384 (2010).

47. Kaikkonen, M. U. et al. Remodeling of the enhancer landscape during macrophage activation is coupled to enhancer transcription. Mol. Cell 51, 310-325 (2013).

48. Koch, F. et al. Transcription initiation platforms and GTF recruitment at tissuespecific enhancers and promoters. Nat. Struct. Mol. Biol. 18, 956-963 (2011).

49. Nakamura, Y. et al. Targeting of super-enhancers and mutant BRAF can suppress growth of BRAF-mutant colon cancer cells via repression of MAPK signaling pathway. Cancer Lett. 402, 100-109 (2017).

50. Babu, D. \& Fullwood, M. J. Expanding the effects of ERG on chromatin landscapes and dysregulated transcription in prostate cancer. Nat. Genet. 49, 1294-1295 (2017).

51. Shen, Y. et al. Recombinant decorin fusion protein attenuates murine abdominal aortic aneurysm formation and rupture. Sci. Rep. 7, 15857 (2017).
52. Dwight, T. et al. TERT structural rearrangements in metastatic pheochromocytomas. Endocr.-Relat. Cancer 25, 1-9 (2018).

53. Northcott, P. A. et al. Enhancer hijacking activates GFI1 family oncogenes in medulloblastoma. Nature 511, 428-434 (2014).

54. Wang, Y. et al. CDK7-dependent transcriptional addiction in triple-negative breast cancer. Cell 163, 174-186 (2015).

55. Baylin, S. B. \& Jones, P. A. A decade of exploring the cancer epigenome - biological and translational implications. Nat. Rev. Cancer 11, 726-734 (2011).

56. Elsasser, S. J., Allis, C. D. \& Lewis, P. W. Cancer. New epigenetic drivers of cancers. Science 331, 1145-1146 (2011).

57. Cole, P. A. Chemical probes for histone-modifying enzymes. Nat. Chem. Biol. 4, 590-597 (2008).

58. Dawson, M. A. \& Kouzarides, T. Cancer epigenetics: from mechanism to therapy. Cell 150, 12-27 (2012).

59. Marks, P. A. \& Xu, W. S. Histone deacetylase inhibitors: potential in cancer therapy. J. Cell. Biochem. 107, 600-608 (2009).

60. Dawson, M. A. et al. Inhibition of BET recruitment to chromatin as an effective treatment for MLL-fusion leukaemia. Nature 478, 529-533 (2011).

61. Delmore, J. E. et al. BET bromodomain inhibition as a therapeutic strategy to target c-Myc. Cell 146, 904-917 (2011).

62. Mertz, J. A. et al. Targeting MYC dependence in cancer by inhibiting BET bromodomains. Proc. Natl Acad. Sci. USA 108, 16669-16674 (2011).

63. Zuber, J. et al. RNAi screen identifies Brd4 as a therapeutic target in acute myeloid leukaemia. Nature 478, 524-528 (2011).

64. LeRoy, G., Rickards, B. \& Flint, S. J. The double bromodomain proteins Brd2 and Brd3 couple histone acetylation to transcription. Mol. Cell 30, 51-60 (2008).

65. Rahman, S. et al. The Brd4 extraterminal domain confers transcription activation independent of pTEFb by recruiting multiple proteins, including NSD3. Mol. Cell. Biol. 31, 2641-2652 (2011).

66. Yang, Z. et al. Recruitment of P-TEFb for stimulation of transcriptional elongation by the bromodomain protein Brd4. Mol. Cell 19, 535-545 (2005).

67. Jang, M. K. et al. The bromodomain protein Brd4 is a positive regulatory component of P-TEFb and stimulates RNA polymerase II-dependent transcription. Mol. Cell 19, 523-534 (2005).

68. Chipumuro, E. et al. CDK7 inhibition suppresses super-enhancer-linked oncogenic transcription in MYCN-driven cancer. Cell 159, 1126-1139 (2014).

69. Trabucco, S. E. et al. Inhibition of bromodomain proteins for the treatment of human diffuse large B-cell lymphoma. Clin. Cancer Res. 21, 113-122 (2015).

70. Valentijn, L. J. et al. TERT rearrangements are frequent in neuroblastoma and identify aggressive tumors. Nat. Genet. 47, 1411-1414 (2015).

71. Weischenfeldt, J. et al. Pan-cancer analysis of somatic copy-number alterations implicates IRS4 and IGF2 in enhancer hijacking. Nat. Genet. 49, 65-74 (2017).

72. Hahn, C. N. et al. Heritable GATA2 mutations associated with familial myelodysplastic syndrome and acute myeloid leukemia. Nat. Genet. 43, 1012-1017 (2011).

73. Ostergaard, P. et al. Mutations in GATA2 cause primary lymphedema associated with a predisposition to acute myeloid leukemia (Emberger syndrome). Nat. Genet. 43, 929-931 (2011).

74. Zhang, $X$. et al. Identification of focally amplified lineage-specific super-enhancers in human epithelial cancers. Nat. Genet. 48, 176-182 (2016).

75. Drier, Y. et al. An oncogenic MYB feedback loop drives alternate cell fates in adenoid cystic carcinoma. Nat. Genet. 48, 265-272 (2016).

76. Lin, C. Y. et al. Transcriptional amplification in tumor cells with elevated c-Myc. Cell 151, 56-67 (2012).

77. Bhagwat, A. S. \& Vakoc, C. R. Targeting transcription factors in cancer. Trends Cancer 1, 53-65 (2015).

78. Nicodeme, E. et al. Suppression of inflammation by a synthetic histone mimic. Nature 468, 1119-1123 (2010).

79. Filippakopoulos, P. et al. Selective inhibition of BET bromodomains. Nature $\mathbf{4 6 8}$, 1067-1073 (2010)

80. Ott, C. J. et al. BET bromodomain inhibition targets both c-Myc and IL7R in highrisk acute lymphoblastic leukemia. Blood 120, 2843-2852 (2012).

81. Di Micco, R. et al. Control of embryonic stem cell identity by BRD4-dependent transcriptional elongation of super-enhancer-associated pluripotency genes. Cell Rep. 9, 234-247 (2014).

82. Dawson, M. A. et al. Recurrent mutations, including NPM1c, activate a BRD4dependent core transcriptional program in acute myeloid leukemia. Leukemia $\mathbf{2 8}$, 311-320 (2014).

83. Garriga, J. \& Grana, X. Cellular control of gene expression by T-type cyclin/CDK9 complexes. Gene 337, 15-23 (2004).

84. Nilson, K. A. et al. THZ1 reveals roles for Cdk7 in co-transcriptional capping and pausing. Mol. Cell 59, 576-587 (2015).

85. Arner, E. et al. Transcribed enhancers lead waves of coordinated transcription in transitioning mammalian cells. Science 347, 1010-1014 (2015). 
86. Core, L. J. et al. Analysis of nascent RNA identifies a unified architecture of initiation regions at mammalian promoters and enhancers. Nat. Genet. 46, 1311-1320 (2014).

\section{ACKNOWLEDGEMENTS}

This work was supported in part by the National Natural Science Foundation of China (81872226, 81402265, 81502346), China Postdoctoral Science Foundation-funded project (2016M592580). We thank various members in Professor Faqing Tang Laboratory for contributions and helpful discussion. We appreciate the contributions and helpful discussion of various members in Clinical Laboratory of Hunan Cancer Hospital \& the Affiliated Cancer Hospital, Central South University.

\section{AUTHOR CONTRIBUTIONS}

All authors reviewed and approved the final manuscript. F.T. contributed to the conception and design of paper. Z.Y. drafted the paper. Y.T. and Y.L. revised the paper.

\section{COMPETING INTERESTS}

The authors declare no competing interests.

\section{ADDITIONAL INFORMATION}

Correspondence and requests for materials should be addressed to F.T.

Reprints and permission information is available at http://www.nature.com/ reprints

Publisher's note Springer Nature remains neutral with regard to jurisdictional claims in published maps and institutional affiliations.

(C) Open Access This article is licensed under a Creative Commons Attribution 4.0 International License, which permits use, sharing, adaptation, distribution and reproduction in any medium or format, as long as you give appropriate credit to the original author(s) and the source, provide a link to the Creative Commons license, and indicate if changes were made. The images or other third party material in this article are included in the article's Creative Commons license, unless indicated otherwise in a credit line to the material. If material is not included in the article's Creative Commons license and your intended use is not permitted by statutory regulation or exceeds the permitted use, you will need to obtain permission directly from the copyright holder. To view a copy of this license, visit http://creativecommons. org/licenses/by/4.0/.

(c) The Author(s) 2020 